\title{
Melanoma Peptide Vaccines: From Predinical Background to Clinical Trials
}

\author{
Jeffrey Weber, MD, PhD
}

\begin{abstract}
Address
University of Southern California/N orris Comprehensive Cancer Center, 1441 Eastlake Avenue, Suite 3447, Los Angeles, CA 90089, USA.

Current Oncology Reports 2000 2:38-47

Current Science Inc. ISSN 1523-3790

Copyright $\odot 2000$ by Current Science Inc.

The discovery that immune T-cells recognize intracellularly processed peptides associated with major histocompatibility locus molecules has revolutionized the cancer vaccine field by providing new reagents for the generation of immune responses against cancer. The cloning of tumor antigen genes has proceded most rapidly in melanoma because of the ease with which melanoma-specific T-cells can be propagated in vitro. The cloning and identification of tumor regression antigens and data from the initial clinical trials with peptides vaccines derived from those antigens are presented here.
\end{abstract}

\section{Introduction}

Eukaryotic cells express thousands of proteins which are degraded intracellularly into peptides via the multisubunit proteosomeand presented on the surface of antigen-presenting cells as antigens for recognition by T-cells in association with class I and II major histocompatibility locus (MHC) molecules. The generation and detection of antigen-specific antitumor immune responses in humans has been revolutionized by the discovery that peptides are the ultimate target for T-cells [1-3]. A number of clinical trials of peptide vacanes with and without adjuvants in patients with metastatic and resected melanoma have been facilitated by the cloning and identification of several dasses of tumor-associated and tumor-specific antigens on melanomas. A number of different classes of defined melanoma antigens are summarized herein. Also provided are descriptions of the available published and unpublished data concerning recent attempts to boost immunity directed against defined melanoma antigens using peptides, al ong with discussion of their clinical significance. Finally, a suggestion for an "optimal" cancer vaccine design is provided in the context of current peptide vaccine efforts in melanoma and other histologies.

\section{Antigens Present on Human Melanomas}

Cancer vaccine development has progressed most rapidly for patients with malignant melanoma, for which compel- ling evidence of immunogenicity exists in humans. Spontaneous regression of primary melanomas is common and can be observed pathologically as well as clinically, and the prognosis of cutaneous melanomas varies directly with the level of Iymphocytic infiltrate [4-5]. Vitiligo is an autoimmune destruction of melanocytes mediated by serologic and T-cell responses that commonly occurs in melanoma patients, especially those who have been treated with interferon- $\alpha$ or interleukin-2 (IL-2), and it is known to correlate with a favorable response to immunotherapy with highdose IL-2 or chemobiotherapy [6-8]. Data from murine experiments and in melanoma patients suggests that both humoral and cellular immune responses to TRP-2, a component of the melanosome, is responsible for vitiligo. Tumor-reactive lymphocytes derived from patient peripheral blood or found to infiltrate metastatic melanoma lesions can be grown in vitro as oligoclonal T-cell lines or clones. The ease with which such melanoma specific T-cell clones can be grown in vitro al lowed investigators in 1991 to describe the cloning of an antigen derived from a mutagenized melanoma cell line which was recognized by T-cells $[12 \cdot, 13]$. This antigen was called MAGE (melanoma antigen-E), and it defined a family of antigens that had not previously been identified. MAGE- 1 and several members of its multigene family were present on a significant proportion of melanoma cell lines and fresh tumors but were also found on a variety of tumors of epithelial and neuroectodermal origin as well as normal testis and placental tissue, but on no other normal tissue [14]. MAGE, GAGE, BAGE, and RAGE-defined families of genes linked to theXchromosome that were found respectively on melanoma, gastrointestinal, breast, and renal-cell tumors, many of which encoded antigens that were recognized by T-cells and defined the "cancer/testis" class of tumor antigens. Between $20 \%$ and $80 \%$ of melanoma lines and fresh tumors were shown to express cancer/testis antigens from these four families.

After the cloning of the first cancer/testis antigens, several groups defined members of a second group of differentiation antigens that were melanosome-related "neoantigens" derived from gene products produced in normal cells. The first melanosome-related antigen cloned was MART-1/Melan A, defined via recognition by cytolytic T-cell (CTL) clones from the peripheral blood of melanoma patients and by lymphocytes infiltrating tumors (TIL). The $T L$ that recognized MART-1 as well as $T L$ from a number of 
other melanoma patients reacted with virtually all melanoma cell lines that expressed HLA-A2, and transfection of the $A 2$ gene into other non-A2 expressing melanoma lines increased their sensitivity to TIL Iysis [15]. These data suggested that MART-1 was a common HLA-A2 restricted mela noma antigen recognized by CTL. MART-1 was expressed by virtually all metastatic melanoma lesions, a majority of cell lines derived from metastatic melanomas, and al so by melanocytes, but not by any other normal tissue The nonamer sequence AAGIGILTV and decamer GAAGIGILTV, representing residues 27-35 and 26-35 of MART-1, respectively, bound most strongly to HLA-A2 [16]. These peptides stimulated the growth of specific CTL from peripheral blood mononuclear cells (PBMC) of melanoma patients and in normal tissue [17]. Multiple restimulations of PBMC with MART-1:27-35 peptide in the presence of IL-2 resulted in MART-1-specific CTL derived from 11 of 12 melanoma patients $[18,19]$. These CTL lysed fresh uncultured melanoma cells and were 100 -fold more lytically active against melanoma cells than TIL grown in high-dose IL-2. The majority of TIL grown from patients with melanoma are capable of recognizing the MART-1:27-35 and 26-35 peptides, and a minority of those TIL cultures induced regression of metastatic melanoma after adoptive transfer with high-dose IL-2 [20]. The repertoire of V $\beta$ T-cell receptor molecules from $T L$ and peripheral blood-derived CTL lines that are MART-1-spedific is quite skewed [21-22].

Peptides derived from MART-1 were eluted from melanoma cells, suggesting that MART-1 is a natural ly occurring antigen on fresh tumors [23]. A protein database analysis demonstrated that sequences conforming to the MART-1 A2 binding motif and possessing features important for CTL recognition occurred frequently in normal and viral proteins [24,25], and that a peptide derived from glycoprotein $C$ of herpes simplex virus could sensitize target cells to lysis by MART-1-specific CTL [26]. These data suggest that epitope mimicry by normal or other commonly occurring proteins may account for the frequency of CTL detected against mel anoma antigens like MART-1.

Greater MART-1 reactive CTL activity was demonstrated in the peri pheral blood of melanoma patients compared with normal subjects, suggesting that a tumor related "priming" effect had occurred [27]. In a clinical study of patients with metastatic melanoma who had lymph nodal disease that was resected, a novel technology using peptide-bound class I tetramers was employed to detect MART1 and tyrosinase-specific CTL in tumor-infiltrating lymph nodal tissue From $0.1 \%$ to $3 \%$ of CD8+lymphocytes infiltrating tumor-involved lymph nodes were MART-1 specific; in contrast, the proportion of MART-1-specific cells in non-tumor-containing lymph nodes was not different from background [28•]. The infiltrating tumor antigenspecific CTL were functional, and they secreted cytokines or could lyse peptide-pulsed targets or antigen-expressing tumor cells. Two subsequent studies using melanoma peptidetetramers for the detection and enumeration of tumor- antigen specific CD8 CTL have suggested that fresh peripheral blood-derived T-cells are deficient in recognition of peptide-bearing target cells and neither upregulate CD69 nor secrete cytokines after exposure to peptide-bearing target cells, whereas viral-specific CTL from the same patient were highly active in the same assays. MART-1-specific CD8+cells in the peripheral blood were shown to be predominantly CD45RA-naïve cells, not CD45RO-activated or memory cells.

The overlapping MART-1 26-35 peptide has been shown to be more immunogenic than the 27-35 epitope, and a single amino acid modification to the 26-35 peptide rendered it a stronger binder to $A 2.1$ and even more immunogenic [28•]. This peptide is a candidate for future clinical vaccine trials. The MART-1 26-35 and 27-35 peptides have been shown to bind to multiple other $A 2$ subtypes with a significant degree of heterogeneity [29,30], as well as allele A45 [31], but no other MART-1 specific peptides have yet been shown to el icit specific immune responses in patients bearing other HLA class I alleles [32].

Antigen pMel17/gp100 was defined through recognition by CTL clones from melanoma patient peripheral blood and by TIL. gp100 is a transmembrane glycoprotein of approximately 100 kilodal tons recognized by the HMB45 and NKI-beteb monoclonal antibodies on melanocytes and melanoma cells [33,34]. CTL clones derived from melanoma patients and TIL grown from a melanoma patient recognized melanoma cells that expressed gp100 in association with HLA-A2.1, but not gp100 negative lines [35•]. Multiple peptides derived from the gp100 sequence that fit the consensus motif for binding to HLA-A2 can be recognized by TIL from melanoma patients, including gp100 209-217 (ILDQVPSFV), gp100 154-162 (TKTWGQYWQV), and gp100 457-466 (LLDGTAATLRL) [36]. A naturally processed gp100 peptide 280-288 (YLEPGPVTA) was defined by elution of peptides from melanoma cells and purification by tandem mass spectroscopy. This peptide redirected HLA-A2 restricted lysis by CTL lines from a melanoma patient [37]. TIL from a melanoma patient al so recognized gp100 280-288. All three gp100 peptides are recognized by self-reactive T- cells, and they bind with moderate or low affinity to HLA A2. This suggests that the gp100-specific TIL or CTL may have escaped from tolerance induction because of their low affinity for TcR (T-cell receptor), whereas the autoreactive CTL that bear high-affinity TCR may be deleted in the thymus. It is of some interest that, in melanoma patients, gp100-specific TIL have been reported to induce regression of metastatic melanoma in patients receiving TIL and IL-2 therapy. Four of a group of 14 TIL that were analyzed were reactive with one or more epitopes of gp100; all four induced tumor regression [35]. Peptides derived from gp100 that are recognized by CTL in association with members of the HLA-A3 superfamily have also been defined, suggesting that the repertoire of HLA haplotypes eligible for vaccination trials with gp100 may be expanded [38,39]. These data al so suggest that gp100 may 
be a promising target for immunotherapy using peptide vaccination strategies and/or adoptive therapy of antigenspecific cytolytic T-cells.

Tyrosinase is a membrane-bound protein involved in the melanin synthesis pathway [40]. Tyrosinase is expressed by virtually all primary cutaneous melanomas and by up to $90 \%$ of metastatic lesions [40]. It encodes several epitope peptides that are presented by HLA-A2 to CTLreactive human melanomas [41]. A peptide derived from tyrosinase, aa 368-376, YMNGTMSQV, was shown to be posttranslationally modified by deamidation of asparagine to aspartic acid resulting in a sequence recognized by human CTL, YMDGTMSQV, known as tyrosinase 368-376 (370D) [42]. The tyrosinase 368-376 (370D) peptide is thought to encode a biological ly important epitope and can induce CTL in vitro (Unpublished data, Weber et al).

TRP-1 (gp75) and TRP-2 aretyrosinase-related proteins that are integral parts of the melanosome apparatus, and they have been shown to be recognized by CTL from patients with melanoma, although these neo-antigens have not yet reached the clinical trial stage [43-45].

Recently, a "cancer-testis" antigen distinct from the MAGE, GAGE, BAGE, and RAGE families, called NY-ESO-1, was discovered. It has the property of eliciting both a strong humoral response but al so encodes epitope peptides recognized by CTL clones and TIL clones from patients with melanoma and breast cancer $[46 \bullet 47]$. The antigen was found to be expressed by a variety of squamous and adenocarcinomas as well as by melanoma, suggesting a wide potential utility.

A final group of melanoma antigens are mutated normal genes that are uniquely present on individual tumors; p16, $\beta$-catenin, and mum-1 are examples [48-50]. Because these genes are unique to individual tumors, their clinical utility for vaccines is limited and will not be pursued in this review.

\section{Clinical Trials with Peptide Vaccines}

Early trials of peptide vaccines for melanoma were performed in patients with metastatic disease to test the toxicity of and immune responses to the vaccine. The peptide vaccines showed few toxicities other than local pain and granuloma formation. Escalating doses of peptides in aqueous solution or combined, as in prior mouse experiments, with incomplete Freund's adjuvant (IFA or Montanide ISA 51, an oil-in-water emulsion) at doses up to 10 mg showed no serious side effects. Initial experiments with the HLA-A1-restricted MAGE-3 peptide EVDPIGHLY given in aqueous solution at low ( 100 to $300 \mu \mathrm{g} /$ injection) doses to 12 patients with metastatic melanoma indicated that six patients were able to complete three injections at monthly intervals, and three of the six had objective clinical responses [51 •]. No evidence of boosted immunity was detected in the peripheral blood. When the same epitope peptide was combined with IFA and administered to patients with resected melanoma, immune responses were detected by CTL assays in five of 16 patients tested at our institution, and delayed-type hypersensitivity (DTH) skin responses to peptide were also observed [52]. No clear correlation was observed in this small trial between immune response by specific cytokine or chromium rel ease after exposure to peptide-pulsed T2-Al cells and time to relapse.

The MART-1 27-35 and 26-35, tyrosinase 1-9 and 368376 , as well as several gp100 peptides ( $280-288$ and $457-$ 467), have been tested al one and in combination in patients with metastatic or resected melanoma. Jaeger et al. [53] immunized six metastatic melanoma patients intradermally with multiple peptides from MART-1, gp100, and tyrosinase in aqueous solution at $100 \mu \mathrm{g}$ each on a weekly basis for four immunizations. These authors observed augmented cytolysis of antigen-restimulated PBMC directed against one, but not necessarily all three, antigens [53]. Three of six patients showed augmented cytolysis to MART-1, and two of six to a tyrosinase peptide, but none of six demonstrated augmented reactivity to gp100 peptides. When granul ocyte-macrophage colony stimulating factor (GM-CSF) was injected at 75 micrograms per dose subcutaneously as an adjuvant treatment for 3 days prior to and 2 days after immunization with multiple antigen peptides, significant boosting of immune reactivity was seen compared with vaccination using peptides al one [54]. Three of three patients showed increased immune reactivity to tyrosinase peptide 1-9, and one of three had increased reactivity to MART-1 26-35. Three of three objective clinical responses were seen, including two partial regressions in involved lymph nodal, cutaneous, and liver lesions, and one complete regression in a patient with subcutaneous disease An infiltrate of CD8 (+) T-cells was observed at vaccination sites after GM-CSF injections.

In subsequent peptide vaccine trials in melanoma, it was shown that six of 26 patients treated with MAGE-1, MAGE-3, MART-1/Melan A and tyrosinase peptides exhibited tumor regression [55]. Disease was stabilized in 22 of 26 evaluable patients with metastatic melanoma. A corre lation was seen between strong MHC class I staining and response; there was al so a correlation between skin/subcutaneous disease, low tumor burden, immuneresponse, and clinical response. The authors argue that the strength of the correlation between clinical response and MHC expression would suggest that MHC staining of tumor by immunohistochemistry should be an el igibility criteria for peptide vaccine trials, which is consistent with other published data. Surprisingly, there was little loss of tumor antigen expression, al though one of 11 patients had cytolytic T-cell responses to MAGE-1/3, eight of 30 to MART-1/Melan A, and six of 30 to tyrosinase peptides.

Cebon et al. [56] immunized patients with metastatic melanoma using intradermally administered MART- $1_{26-35}$ peptidewith IL-12 in increasing doses given either subcutaneously or intravenously. The main toxicity of IL-12 by either routewas flu-like symptoms. Of the first 15 patients, one complete 
regression (CR), one partial regression (PR), and one mixed response were noted. Immune assays for T-cell generation included delayed type hypersensitivity, which was seen in patients with or without IL-12. Positive CTL assays were seen in patients with evidence of dinical benefit, but not in patients without regression. Scheibenbogen et al. [57] immunized 18 patients with four HLA dass I restricted tyrosinasepeptides, 16 with metastatic melanoma, and two adjuvant patients without evidence of disease Increased CTL activity was observed in four of 15 patients, two adjuvant patients, and two patients with evidence of tumor regression. As in the trial by Cebon et al. [56] and in other trials, patients with progressivediseasedid not show boosted antigen-spedific immunity.

A number of small pilot studies have been conducted at the National Cancer Institute of the US National Institutes of Heal th in which patients with metastatic melanoma received multiple subcutaneous injections of a single peptide emulsified with IFA at 3-week intervals. MART-1 27-35, gp100 209$217,154-162$, and 280-288 have been used in these trials [58,59]. In one study, escalating doses of the gp100 209 (ITDQVPSFY), 280 (YLEPGPVTA), or 154 (KTWGQYWQV) peptides at doses from 1 to $10 \mathrm{mg}$ were administered subcutaneously every 3 weeks with IFA [60]. Immune assays were performed using the above "native" peptides for antigenic stimulation and substituted 209-2M (IMDQVPFSY) and $280-9 \mathrm{~V}$ (YLEPGPVTV) peptides. Ninety percent to $100 \%$ of patients had strong evidence of boosted immune reactivity postvaccination as shown by an assay in which release of interferon- $\gamma$ from PBMC restimulated one to three times in the presence of IL-2 and peptide antigen was measured by enzyme-linked immunosorbent assay (ELISA). Seven of seven patients had boosted gp100 reactivity postvaccine after only one restimulation with the $209-2 \mathrm{M}$ peptide, and five of six were boosted with one restimulation with the $280-9 \mathrm{~V}$ peptide Higher rel ease of cytokine was seen after four immunizations than in two immunizations in most patients, and a greater level of reactivity was observed when substituted peptides were used in assays, in contrast with "native" peptides. Boosted cytokine rel ease was shown to correlate with cytolytic responses. When tumor cell lines expressing the correct $\mathrm{MHC}$ restriction el ement and antigen were used as a stimulator in cytokine re ease assays, lower levels of cytokine were observed compared with $\mathrm{T} 2$ cells pulsed with the rele vant peptide This suggests that peptide density on the target was important for recognition by effector cells in PBMC. Objective partial and complete remissions were uncommon, with one of 20 patients having a complete regression. No dear correlation was observed between the level of immune responseand therare clinical responses, so that no statement about clinical benefit could be made In a second study of escal ating doses of the MART-1 27-35 peptide administered subcutaneously every 3 weeks with IFA, 15 of 16 patients had evidence of boosted immunity directed against the "native" MART-1 27-35 peptide, with increased reactivity after four vaccinations compared with fewer vaccinations [61]. No objective clinical responses were seen.
At the University of Southern California/Norris (UC/ Norris), we have treated 25 melanoma patients with highrisk resected stage III/IV disease with increasing doses of the MART-1 27-35 peptide emulsified with IFA every 3 weeks subcutaneously [62]. Toxicity consisted of local inflammation and granuloma formation, and low-grade fevers, fatigue, headache, and myalgias were common. Ten of 22 patients had evidence of boosted immunity by cytokine release assays, and a correlation was observed between the absolute level of interferon- $\gamma$ rel eased after multiple restimulations of peptide-pulsed patient PBMC postvaccination and time to relapse, with a P-value of 0.003. Thecorrelation of immune reactivity with time to relapse suggests that a positive response to peptide vaccination may incur clinical benefit. The data suggest that patients mount an immune response to flutamide (FLU-M) before and after vaccination and that the development of MART-1-specific CTL is antigen-specific, because MART-1-stimulated CTL do not react with FLU targets, and viceversa. All nine of the relapses and all three deaths have occurred in the minimal response or no response group thus far, supporting the idea that devel opment of an immune response directed against MART-1 is clinically beneficial.

The anchor amino acids that form hydrogen bonds between epitope peptides and class I MHC molecules can be modified to strengthen their binding, resulting in greater immunogenicity in vitro and in vivo. When such heteroclitic peptides derived from gp100 were used to restimulate PBMC from patients with metastatic melanoma immunized with a wild-type gp100 peptide, immune reactivity was detected with greater frequency and sensitivity [60]. Patients immunized with the heteroclitic gp100 peptide demonstrated a higher level of immune reactivity compared with those immunized with the wildtype peptide The MART-1 27-35 peptide has been shown to be "naturally" processed and is immunodominant; however, the MART-1 26-35 peptide has been shown to be a better $\mathrm{MHC}$ binder and to be moreimmunogenic in vitro [63] and was more effective at detection and quantitation of MART-1-specific CTL in a flow cytometry assay when used for the generation of $\mathrm{MHC} /$ peptide tetramers [28•]. Heteroclitic gp100 peptides combined with IFA have been used to immunize patients with metastatic melanoma. More than $90 \%$ of the patients in one vaccine study had evidence of boosted immunity detected after one restimulation in vitro, suggesting that the substituted peptide was more immunogenic than "natural" peptide in vivo [64•. When the same substituted gp100 peptide was injected and followed within several days by high-dose intravenous IL2 , surprisingly, no detectable augmented immune response was observed, but 13 of 31 patients had an antitumor response, including 12 PRs and one CR, for an overall response rate of $42 \%$, significantly greater than rates observed with IL-2 alone (15\% to $20 \%)[60,64 \cdot, 65,66]$.

The utility of a peptide vaccination added to high- dose IL-2 as an "adjuvant" is currently being explored in two 


\begin{tabular}{|c|c|c|c|c|c|c|c|c|c|}
\hline \multirow[t]{2}{*}{$\begin{array}{l}\text { Patients, } \\
\text { n/PRE/POST }\end{array}$} & \multicolumn{3}{|c|}{$\begin{array}{l}\text { Cytokine release/ } \\
\text { FLU stim* }\end{array}$} & \multicolumn{3}{|c|}{$\begin{array}{l}\text { Cytokine release/ } \\
\text { gp100 stim }\end{array}$} & \multicolumn{3}{|c|}{$\begin{array}{c}\text { Cytokine release/ } \\
\text { tyr stim }\end{array}$} \\
\hline & T2 FLU & T2 gp100 & T2 tyr & T2 FLU & T2 gp100 & T2 tyr & T2 FLU & T2 gp100 & T2 tyr \\
\hline $\begin{array}{l}10236 \text { PRE } \\
10313 \text { POST }\end{array}$ & $\begin{array}{l}860 \\
346\end{array}$ & $\begin{array}{c}0 \\
96\end{array}$ & $\begin{array}{c}222 \\
14\end{array}$ & $\begin{array}{l}0 \\
0\end{array}$ & $\begin{array}{c}0 \\
830\end{array}$ & $\begin{array}{c}40 \\
0\end{array}$ & $\begin{array}{c}12 \\
0\end{array}$ & $\begin{array}{c}0 \\
190\end{array}$ & $\begin{array}{c}370 \\
1181\end{array}$ \\
\hline
\end{tabular}

large multicenter trials. At our institution, the heteroclitic gp100 209-217 (210M) and tyrosinase 368-376 (370D) peptides emulsified in IFA with or without IL-12 as an adjuvant are being examined in a randomized phasell trial in patients with high-risk resected stage III/IV melanoma. Immune response is one end point, and time to relapse is the clinical end point. Strong DTH skin test responses to gp100 but not to tyrosinase have been observed in 20 of 24 patients, and augmented immune responses to both gp100 and tyrosinase have been observed in 19 of 24 patients, suggesting that multiple melanoma peptides injected simultaneously with adjuvant result in boosted immunity to both antigens in virtually all patients with resected highrisk melanoma (Unpublished data, Weber et al.). Whether IL-12 administration results in augmented skin test reactivity or increased antigen-specific immune responses measured by cytokine release or tetramer assays awaits a more detailed analysis (Table 1).

In one study, an HLA-A2.1 restricted tyrosinase peptide combined with adjuvant QS-21 was used to vaccinate patients with metastatic melanoma, and no tumor responses were seen, al though in two of nine patients there was evidence of boosted tyrosinase-specific immunity [67]. Toxicity was modest, with fevers and local injection site pain as the only side effects.

Peripheral blood mononuclear antigen-presenting cells prepared by purifying adherent cells and exposing them to $1000 \mathrm{U} / \mathrm{mL}$ of GM-CSF for 7 days were pul sed with the MAGE-1 Al restricted peptide EVDPIGHLY and injected into HLA-Al patients with metastatic melanoma [68]. In a small pilot trial, T Iymphocytes were grown from the vaccination site of two patients and found to be MAGE1 reactive No significant toxicity was observed in this trial. In one patient, T lymphocytes grown from a peripheral tumor were found to be MAGE-1-specific and could lyse autologous tumor cells. When a DTH assay was performed with peptide-pulsed, antigen-presenting cells (APCs) injected intradermally, a CD8 and CD4 infiltrate was seen, but no patients had an antitumor response. It was suggested that growth of vaccine site infiltrating lymphocytes $(\mathrm{VIL})$ and the demonstration that they were tumor specific constituted a surrogate assay for immune response

Gajewski et al. [69] took a different approach to peptide based therapy, and treated 15 metastatic melanoma patients with subcutaneous Melan A/MART-1 peptidepulsed peripheral blood cells with rhul L-12 in escalating doses subcutaneously every other day for three doses after each cell injection. OnePR, two MRs, and threepatients with stable disease were observed, with minimal toxicity. Antigen-specific immune reactivity was augmented in eight of 13 patients by the peptide-pulsed cell injections. These responses were directed against MAGE-3 and MelanA/MART-1.

Rosenberg et al. [70] used adenoviral vectors encoding the cDNAs for gp100 and MART-1 to immunize patients with metastatic melanoma. They performed a phase I trial of escalating doses of recombinant virus from $10^{7}$ to $10^{11}$ particles per injection, given either intradermally or subcutaneously, either alone or with the addition of high-dose IL-2. Thirty-six patients received the MART-1 adenoviral vector, and 18 received the gp100 construct. Immunizations were given four times at monthly intervals. Mild and transient erythema at injection sites was the only side effect observed. One of 16 patients who were given the MART-1 vector had a complete response, but none of six patients who received the gp100 adenovi rus al one responded. Of the 20 patients who were given IL-2 plus the MART-1 vector, there were two PRs and two CRS. No consistent augmentation of antigen-specific T-cell reactivity was observed in any subgroup of patients, in contrast with the significant reactivity seen with the use of peptides with IFA by the same investigators. Only five of 23 patients had augmented MART-1 immunity detected by release of cytokines by restimulated PBMC, and none of 6 had gp100 boosting; however, 34 of 45 patients sera-tested for adenoviral antibodies showed significant increases in antiviral IgG. These data suggested to the investigators that adenoviral serologic reactivity might limit the utility of engineered adenoviral vectors as vaccines, and they concluded that current recombinant adenoviral vaccines were not superior to peptide-based immunizations.

Dendritic cells (DC) serve as "nature's adjuvant." They are antigen-presenting cells that can prime naïveT-cells, and they are potent stimulators of cytolytic and helper T-cell immunity [71-75]. Dendritic cells pulsed with melanoma antigen peptides can stimulate the devel opment of antigenspecific CTL in vitro more potently than peptide-pulsed PBMC or macrophages [76]. DC have been prepared from the adherent peripheral blood monocytes of patients by 


Table 2. Immune Response to gp100/tyrosinase Peptide-pulsed Dendritic Cell Therapy
\begin{tabular}{|lccccccccc|}
\hline $\begin{array}{l}\text { Patients, } \\
\text { n/PRE/POST }\end{array}$ & $\begin{array}{c}\text { Cytokine release/ } \\
\text { FLU stim }\end{array}$ & $\begin{array}{c}\text { Cytokine release/ } \\
\text { gp100 stim }\end{array}$ & $\begin{array}{c}\text { Cytokine release/ } \\
\text { tyr stim }\end{array}$ \\
\hline & T2 FLU & T2 $\mathbf{~ g p 1 0 0 ~}$ & T2 tyr & T2 FLU & T2 $\mathbf{~ g p 1 0 0 ~}$ & T2 tyr & T2 FLU & T2 $\mathbf{~ g p 1 0 0 ~}$ & T2 tyr \\
\hline 10236 PRE & $\mathbf{6 7 0}$ & 0 & 0 & 0 & 88 & 0 & 0 & 0 & 0 \\
10313 PO ST & $\mathbf{1 1 4 8}$ & 0 & 0 & 0 & $\mathbf{1 8 2 2}$ & 0 & 0 & 0 & $\mathbf{9 1 9}$ \\
\hline FLU-flutamide; Stim- stimulation; Tyr- tyrosinase. \\
*Antigen-specific responses are indicated by boldface type.
\end{tabular}

growth in IL-4 and GM-CSF $[77,78]$ and pulsed with multiplemelanoma peptides. In vitro, DC pulsed with melanoma peptides can stimulate the development of potent antigenspecific CTL responses [79]. These DC have been injected subcutaneously and intra-lymph-nodally in patients with metastatic melanoma, and they have been shown to induce antigen-specific immune reactivity and dinical responses. In one published study, onecompleteremission and three partial remissions were observed in patients treated with multiple peptide-pulsed monocyte-derived DC without evidence of significant toxicity [80•]. At our institution, we have treated 16 melanoma patients with intravenous injections of monocyte-derived DC pulsed with multiple heteroclitic melanoma antigen peptides from gp100 and tyrosinase, and we have seen objective clinical responses and boosting of immunity in responding patients, as shown by the immune response assay data summarized in Table 2 . These findings indicate that tyrosinase and gp100-specific CTL cytokine release were observed from a responding patient's PBMC that were restimulated once with rel evant peptides after immunization with peptide-pulsed DC. In contrast with patients who receive peptides with IFA, no peptiderelated DTH skin test reactivity has been observed in patients treated with peptidepulsed DC.

The MART-1, gp100, and tyrosinase antigens are all expressed by normal melanocytes [73-75], and the use of neo-antigen peptide vaccines has the potential to induce autoimmune reactions. It is not known whether normal melanocytes effectively present the MART-1, gp100, or tyrosinase epitope peptides to T-cells in vivo. Previous clinical experience with the adoptive transfer of large numbers of CTL that were highly MART-1-or gp100-reactive and mediated regression of tumor did not indicate the onset of any autoimmune damage to skin, brain, inner ear, and retina, where mel anocyte lineage cells are located. Pati ents with metastatic or resected melanoma who received MART1, gp100, or tyrosinase peptide vaccines at our institution or in studies described in the published literature did not demonstrate any evidence of ocular or other toxicity (Unpublished data, Weber et al.) $[60,63]$. One patient of 75 who received a gp100/tyrosinase vaccine at USC/Norris showed evidence of vitiligo. No ocular problems were observed, nor was there any evi dence of autoi mmune pathology occurring in any patients on our MART-1, gp100, or tyrosinase peptide trials with median follow-ups of greater than 16 months.

One potential pitfall of any antigen-specific immunization strategy is immunosel ection in vivo after peptide vaccination [81-89]. Reverse transcriptase-polymerase chain reaction (RT-PCR) analysis has shown that MART-1 mRNA is present in virtually $100 \%$ of metastatic melanoma lesions, yet immunohistochemical staining has shown that there is considerable heterogeneity in MART-1, with $60 \%$ to $90 \%$ of all cells staining positively [83-89]. In one study, del etion of MART-1 expression and of TAP transporter expression rendered cells transparent to CTL recognition, suggesting that loss of MART-1 may be a mechanism for immune evasion [88]. Two patients who were repeatedly immunized with MART-1, gp100, and tyrosinase peptides in aqueous solution intradermally had tumors biopsied prior to and after vaccination. One patient developed MART-1 reactive T-cells in the peripheral blood, but a biopsy of a progressing lesion showed loss of MART-1 expression. Another patient had an initial biopsy showing expression of all three antigens, but after immunization and development of tyrosinsase-specific reactivity, a progressing tumor expressed gp100/MART-1 but not tyrosinase [89]. A significant level of antigenic heterogeneity occurs within lesions, as well as complete loss of antigen expression on individual lesions [72-79,80 •,81-87]. These data suggest that a successful vaccine would require multiple antigenic peptides to minimize the consequence of loss of any individual antigen.

\section{Functional Analyses of Antigen-specific T-cells in Melanoma Patients}

Functional defects in melanoma-specific T-cells from peripheral blood and tumor-bearing lymph nodes of patients with metastatic melanoma may render them ineffective. Romero et al. [28 •] showed by tetramer staining that MART-1-specific antigen-experienced cells were found in tumor-infiltrated lymph nodes, but not in normal Iymph nodes from the same patients. In a subsequent study, these authors demonstrated that peripheral bloodderived and lymph node-derived MART-1-specific T-cells were predominantly CD45 RA-positive, suggesting that they retained a naïve phenotype [90]. Lee et al. [91 •] iso- 
lated tyrosinase and MART-1-specific tetramer-positive cells from patients with metastatic melanoma and showed that antigen-specific activation of CD69 was deficient on those cells. Sorted antigen-specific tetramer-positive cells from fresh blood did not lyse antigen-expressing targets, nor did they release cytokines unless cultured in the presence of IL-2. These data support and are consistent with experiments performed by several groups, including Finke et al. [92], who showed that circulating total peripheral blood T-cells from patients with melanoma were deficient in TcR transduction pathways [93,94]. These results indicate that peptide and antigen-specific vaccine therapy may certainly augment T-cell responses to high levels, but that functional T-cell assays should be part of any measure of immune response in melanoma vaccine trials. Further efforts to develop strategies for overcoming immune suppression in melanoma are critically important for the successful devel opment of melanoma vaccines.

\section{Condusions}

One of the biggest problems in the cancer vaccine field today is the abundance of good ideas compared with a modest number of patients who would beappropriatecandidates for vaccine trials. Good-prognosis mel anoma patients with metastatic stage IV disease (M1a or M1b) or resected stage IV disease who are rendered disease-free would be logical candidates for vaccine trials because the clinical end points of tumor response and survival or time to relapse would be short. Such patients, however, are few. A second critical issue is the lack of a good surrogate laboratory assay for a successful immune response. Limiting dilution analyses using chromium rel ease assays have been the immunologic "gold standard" for the enumeration of CTL precursors, but this intervention is labor intensive and difficult to perform. Bulk CTL cytokine rel ease assays, enzyme-linked immunospot (ELISPOT) assays, and staining for intracellular cytokines are simpler and more reproducible, but they generally require restimulation of T-cells in vitro for 1 to 7 days, obscuring and artificially amplifying T-cell responses. Tetramer assays can be performed on fresh blood cells and may allow a functional analysis of tumor antigen-specific T-cells from fresh blood without the complications of in vitro restimulation and exposure to cytokines. Tetramer assays could be combined with intracellular cytokine staining and T-cell receptor variable chain analysis to yield important information about the specificity and function of antigen-specific CTL. Peptides with adjuvants or those that are pulsed onto dendritic cells, DNA plasmids encoding peptide mini-genes, or whole antigens and viral vectors encoding peptides/whole antigens are all promising candidates that must be tested.
Dose, schedule, route, and type of adjuvant are all variables that must be worked out in patients in small phase II pilot trials that should be devised based on the best information that we have from mouse models and using the best available immune assay.

Although achieving a clinical response to a particular vaccine regimen in patients with measurable metastatic mel anoma is a desirable standard, the significant tumor burden and well-documented immune suppression present in many patients with bulk metastatic melanoma suggest that this group is not the ideal population in which to test new vaccines. However, it should be accepted that any phase II cancer vaccine trials must incorporate an end point of clinical benefit that should be correlated with assays of immune response. I propose that future vaccine trials be performed in patients with resected high-risk melanoma who have a greater than $50 \%$ chance of relapse and death from metastatic disease, but a mi croscopic disease burden. The dinical end point would be time to relapse, which should be correlated with any immune response assay. Although immune assays with restimulated PBMC have been used, new assays employing intracellular cytokine release and class I peptide tetramer binding combined with spectra-type TcR analysis and followed by multicolor flow cytometry may offer a more sensitive and accurate assessment of antigen-specific reactivity with fresh peripheral blood cells.

An "optimal" peptide vaccine trial that I would propose would include HLA-A2.1 patients who have resected stage IV melanoma and are rendered free of disease. Patients with stage III disease and two or more pal pable lymph nodes, extracapsular extension, or in-transit metastases would also be included because of their poor outcome with surgery al one and the modest clinical benefit shown with interferon- $\alpha$. Multiple heteroditic peptides from tyrosinase, gp100, MART-1, and gp75, at a dose of 300 to $1000 \mu \mathrm{g}$ for each peptide, would be included with a pan-HLA-DR peptide and IFA as the adjuvant. Treatment with Flt-3 ligand for 7 days in the limb to be immunized would precede each injection, and a single dose of IL-12 would be given intradermally after vaccination. Several days later, low doses of IL-2 at "immune reconstituting" doses of $1-3 \mathrm{mU} / \mathrm{M}^{2}$ would be given subcutaneously twice daily for 5 days after each vaccination. A total of eight to ten injections would be given over a period of 6 to 12 months at increasing intervals of time The clinical end points would be time to relapse and overall survival. The immune end points would be tetramer and cytokine rel ease assays on PBMC isolated from fresh blood and propagated for 24 to 48 hours ex vivo in the presence of cytokines but without antigen. A similar trial has been proposed to begin at our institution in the near future 
References and Recommended Reading Recently published papers of particular interest have been highlighted as:

- Of importance

-. Of major importance

1. Townsend ARM, Gotch RM, DaveyJ: Cytotoxic cells recognize fragments of the influenza nucleoprotein. Cell 1985, 42:457-467.

2. Townsend ARM, Rothbard J, Gotch FM, et al.: The epitopes of influenza nucleoprotein recognized by cytotoxic $T$ lymphocytes can be defined with short synthetic peptides. Cell 1986, 44:959-968.

3. Maryanski JL, Paola P, Coradin G, et al.: H-2 restricted cytotoxic $T$ cells specific for HLA can recognize a synthetic HLA peptide. Nature 1986, 324:578-579.

4. Clark WH, Elder DE, Guerny DE, et al.: A model predicting survival in stage I melanoma based on tumor progression. J N atl Cancer Inst 1989, 81:1893-1898

5. Paladugu RR, Yonemoto RH: Biologic behavior of thin melanomas with regressive changes. Arch Surg 1983, 118:41-45.

6. Bystryn JC, Rigel D, Friedman RJ, Kopf A: Prognostic signficance of hypopigmentation in malignant melanoma. Arch D ermatol 1987, 123:1053-1055.

7. Richards JM, Mehta M, Schroeder, et al.: Sequential chemoimmunotherapy for metastatic melanoma. J Clin O ncol 1992, 9:1152-1160.

8. Nordlund JJ, Kirkwood JM, Forget BM, et al.: Vitiligo in patients with metastatic melanoma: a good prognostic sign. J Am Acad D erm 1983, 9:689-697.

9. Anichini A, Mazzocchi A, Fossatti G, Parmiani G: Cytotoxic T lymphocyte clones from peripheral blood and from tumor sites detect intra-tumoral heterogeneity of melanoma cells: analysis of specificity and mechanisms of interaction. J Immunol 1989, 142:3692-3701.

10. Wolfel T, Klehmann E, Muller C, et al.: Lysis of human melanoma cells by autologous cytolyitc $T$ cell clones: identification of human histocompatibility leucocyte antigen A2 as a restriction element for three different antigens. J Exp M ed 1989, 170:797-805.

11. Topalian SL, Solomon D, Rosenberg SA: Tumor specific cytolysis by lymphocytes infiltrating human tumors. J Immunol 1989, 142:3714-3725.

12. - Van Der Bruggen P, Traversari C, Chomez P, et al.: A gene encoding an antigen recognized by cytolytic $T$ lymphocytes on a human melanoma. Science 1991, 254:1643-1647.

The very first report of the cloning of a human geneencoding a tumor antigen recognized by T-cells: MAGE-1.

13. Traversari $C$, van der Bruggen $P$, Luescher I, et al.: A nonapeptide encoded by human gene MAGE-1 is recognized on HLA-A1 by cytolytic T lymphocytes directed against tumor antigen MZ2-E. J Exp M ed 1992, 176:1453-1457.

14. Gauler $B$, van den Eynde $B$, van der Bruggen $P$, et al.: Human gene MAGE-3 codes for an antigen recognized on melanoma cells by autologous lymphocytes. J Exp M ed 1994, 179:921-929.

15. Kawakami $Y$, Eliyahu S, Delgado $C$, et al.: Cloning of the gene coding for a shared melanoma antigen recognized by autologous T cells infiltrating into tumor. Proc $N$ atl Acad Sci U S A 1994, 96:3515-3519.

16. Coulie PG, Brichard V, Van Pel A, et al.: A new gene coding for a differentiation antigen recognized by autologous cytolytic T lymphocytes on HLA-A2 melanomas. J Exp M ed 1994, 180:35-42.

17. Kawakami Y, Eliyahu S, Sakaguchi K, et al.: Identification of the immunodominant peptides of the MART-1 human melanoma antigen recognized by the majority of HLA-A2restricted tumor infiltrating lymphocytes. J Exp M ed 1994, 180:347-352.

18. Stevens E, Jacknin L, Robbins PF, et al.: The generation of tumor specific cytotoxic lymphocytes from melanoma patients using peripheral blood stimulated with allogeneic melanoma tumor cell lines: fine specificity and MART-1 melanoma antigen recognition. J Immunol 1995, 154:762-0767.
19. Romero P, Gervois N, Schneider J, et al.: Cytolytic T lymphocyte recognition of the immunodominant HLA-A*0201-restricted Melan-A/MART-1 antigenic peptide in melanoma. J Immunol 1997, 159:2366-2374.

20. Rivoltini L, Kawakami Y, Sakaguchi K, et al.: Induction of tumor reactive CTL from peripheral blood and tumorinfiltrating lymphocytes of melanoma patients by in vitro stimulation with an immunodominant peptide of the human melanoma antigen MART-1. J Immunol 1995, 154:2257-2265.

21. Cole DJ, Weil DP, Shilyansky J, et al.: Characterization of the functional specificity of a cloned T-cell receptor heterodimer recognizing the MART-1melanoma tumor antigen. Cancer Res 1995, 55:748-752.

22. Sensi M, Salvi S, Castelli C, et al.: T cell receptor structure of autologous melanoma reactive cytotoxic $T$ lymphocyte (CTL) clones: tumor infiltrating lymphocytes overexpress in vivo the TCR chain sequence used by an HLA-A2 restricted and melanocyte lineage-specific CTL clone. J Exp.M ed 1993, 178:1231-1248.

23. Hunt DF, Henderson RA, Shabanowitz J, et al.: Chracterization of peptides bound to the class I molecule HLA-A2.1 by mass spectrometry. Science 1992, 255:1261-1264.

24. Storkus WJ, Zeh HJ, Maeurer MJ, et al.: Identification of human melanoma peptides recognized by class I restricted tumor infiltrating lymphocytes. J I mmunol 1993, 151:3719-3726.

25. Cox AL, Skipper J, Chen $\mathrm{Y}$, et al.: Identification of a peptide recognized by five melanoma-specific human cytotoxic T cell lines. Science 1994, 264:716-719.

26. Loftus DJ, Castelli C, Clay TM, et al.: Identification of epitope mimics recognized by CTL reactive to the melanoma/ melanocyte-derived peptide MART-1(27-35). J Exp M ed 1996, 184:647-657.

27. Marincola FM, Rivoltini L, Salgaller ML, et al.: Differential anti-MART-1/MelanA CTL activity in peripheral blood of HLA-A2 melanoma patients in comparison to healthy donors: evidence of in vivo priming by tumor cells. J Immunother 1996, 19:266-277.

28. - Romero $P$, Dunbar PR, Valmori $D$, et al.: Ex vivo staining of metastatic lymph nodes by class I major histocompatibility complex tetramers reveals high numbers of antigenexperienced tumor specific cytolytic T lymphocytes. J Exp M ed 1998, 188:1641-1650.

The first report of the presence of tumor-specific memory T-cells in lymph nodes draining tumors in patients with melanoma.

29. Fleischhauer K, Tanzarella S, Wallny HJ, et al.: Multiple HLA-A alleles can present an immunodominant peptide of the human melanoma antigen Melan-A/MART-1 to a peptide-specific HLA-A* $0201+$ cytotoxic T cell line. J Immunol 1996, 157:787-797.

30. Rivoltini L, Loftus DJ, Barracchini K, et al.: Binding and presentation of peptides derived from melanoma antigens MART-1 and glycoprotein-100 by HLA-A2 subtypes. Implications for peptide-based immunotherapy. J Immunol 1996, 156:3882-3891.

31. Schneider J, Brichard V, Boon T, et al.: Overlapping peptides of melanocyte differentiation antigen Melan-A/MART-1 recognized by autologous cytolytic T lymphocytes in association with HLA-B45.1 and HLA-A2.1. J N atl Inst Cancer 1998, 75:451-458.

32. Marincola FM: Stringent allele/epitope requirements for MART-1/Melan A immunodominance: implications for peptide-based immunotherapy. J Immunol 1998, 161:877-889.

33. Jimenez M, Maloy WL, Hearing VJ: Specific identification of an authentic clone for mammalian tyrosinase $1989 \mathrm{~J} \mathrm{Biol.}$ Chem 1989. 264:3397-3403.

34. Adema GJ, DeBoer AJ, Vogel AM, et al.: Molecular characterization of the melanocyte lineage specific antigen gp100. J Biol Chem 1994, 269:20126-20133. 
35. - Bakker ABH, Schreurs WJ, deBoer AJ, et al.: Melanocyte lineage specific antigen gp100 is recognized by melanoma-derived tumor-infiltrating lymphocytes. J Exp M ed 1994, 179:1005-1011. This report describes a new human melanoma tumor antigen recognized by T-cells - gp100 - which is the glycoprotein recognized by the HMB-45 antibody.

36. Kawakami Y, Eliyahu S, Delgado C, et al.: Identification of a human melanoma antigen recognized by tumor-infiltrating lymphocytes associated with in vivo tumor rejection. Proc Natl Acad Sci U S A 1994, 91:6458-6462.

37. Bakker $A B H$, Schreurs MWJ, et al.: Identification of a novel peptide derived from the melanocyte specific gp100 antigen as the dominant epitope recognized by an HLA-A2.1 restricted anti-melanoma CTL line Int J Cancer 1995, 62:97-102.

38. Skipper JC, Kittlesen DJ, Hendrickson RC, et al.: Shared epitopes for HLA-A3 restricted melanoma-reactive human cytolytic T Iymphocytes include a naturally processed epitope from pMel17/gp100. J Immunol 1996, 157:5027-5033.

39. Kawashima I, Tsai V, Southwood S, et al.: Idenitification of gp100-derived melanoma-specific cytolytic T lymphocyte epitopes restricted by HLA-A3 supertype molecules by primary in vitro immunization with peptide-pulsed dendritic cells. Int J Cancer 1998, 78:518-524.

40. Brichard V, Van Pel A, Wolfel T, et al.: The tyrosinase gene encodes for an antigen recognized by autologous cytolytic T lymphocytes on HLA-A2 melanomas. J Exp M ed 1993, 178:489-495.

41. Wolfel T, Van Pel A, Brichard V, et al.: Two tyrosinase nonapeptides recognized on HLA-A2 melanomas by autologous cytolytic T lymphocytes. Eur J Immunol 1994, 24:759-764.

42. Skipper JCA, Hendrickson RC, Gulden PH, et al.: An HLA-A2 restricted tyrosinase antigen on melanoma cells results from post-translational modification and suggests a novel processing pathway for membrane proteins. J Exp M ed 1996, 183:527-534.

43. Wang RF, Robbins PF, Kawakami Y, et al.: Identification of a gene encoding a melanoma tumor antigen recognized by HLA-A31 restricted tumor-infiltrating lymphocytes. J Exp M ed 1995, 181:799-806.

44. Wang R-F, Parkhurst MR, Kawakami Y, et al.: Utilization of an alternative open reading frame of a normal gene in generating a human cancer antigen. J Exp M ed 1996, 183:1131-1138.

45. Wang R-F, Appella E, Kawakami Y, et al.: Identification of TRP-2 as a human tumor antigen recognized by cytotoxic T lymphocytes. J Exp M ed 1996, 184:2207-2214.

46. - Jaeger E, Chen Y-T, Drijfhout JW, et al.: Simultaneous humoral and cellular immune response against cancer testis antigen NY-ESO-1: definition of human histocompatibility leukocyte antigen (HLA)-A2 binding peptide epitopes. J Exp M ed 1998, 187:265-274.

This report describes the cloning and definition of a new cancer testis antigen that is present on many tumors and melanomas and that can elicit T-cell and antibody responses.

47. Wang R-F, Johnston SL, Zeng G, et al.: A breast and melanomashared tumor antigen: $T$ cell responses to antigenic peptides translated from different open reading frames. 1998 J Immunol 1998, 161:3596-3606.

48. Wolfel T, Hauer M, Schneider J, Serrano M, et al.: A p16INK4ainsensitive CDK4 mutant targeted by cytotoxic $T$ lymphocytes in a human melanoma. Science 1995, 269:1281-1285.

49. Robbins PF, El-Gamil M, Li YF, et al.: A mutated b-catenin gene encodes a melanoma specific antigen recognized by tumorinfiltrating lymphocytes. J Exp M ed 1996, 183:1185-1192.

50. Coulie PG, Lehmann F, Lethe B, et al.: A mutated intron sequence codes for an antigenic peptide recognized by cytolytic T lymphocytes on a human melanoma. Proc N atl Acad Sci U S A 1995, 92:7976-7980.

51. - Marchand M, Weynants P, Rankin E, et al.: Tumor regression responses in melanoma patients treated with a peptide encoded by MAGE-3. Int J Cancer 1995, 63:883-885.

The first report of clinical benefit in patients treated with a peptide vaccine Surprisingly, no detectable T-cell responses were observed in responding patients.
52. Weber JS, Hua FL, Spears L, et al.: A Phase I trial of a H LA-A1 restricted MAGE-3 epitope peptide with incomplete Freund's adjuvant in patients with resected high-risk melanoma. J Immunother 1999, in press.

53. Jaeger E, Bernhard H, Romero P, et al.: Generation of cytotoxic $T$ cell responses with synthetic melanoma-associated peptide in vivo: implications for tumor vaccines with melanomaassociated antigens. Int J Cancer 1996, 66:162-170.

54. Jaeger E, Ringhoffer M, Dienes H-P, et al.: Granulocyte macrophage colony stimulating factor enhances immune responses to melanoma associated peptides in vivo. Int J Cancer 1997, 67:54-62.

55. Marchand M, van Baren N, Weynants P, et al.: Tumor regressions observed in patients with metastatic melanoma treated with an antigenic peptide encoded by gene MAGE-3 and presented by HLA-A1. Int J Cancer 1999, 80:219-230.

56. Cebon JS, Jaeger E, Gibbs P, et al.: Phase I studies of immunization with Melan-A and IL-12 in HLA-A2 positive patients with stage III and IV metastatic melanoma [abstract]. Proc ASCO 1999, 18:434A.

57. Scheibenbogen $C$, Schmittel A, Keiholz U, et al.: Vaccination with tyrosinase peptides and GM-CSF in metastatic melanoma Proc ASCO 1999, 18:436A.

58. Salgaller MM, Marincola FM, et al.: Immunization against epitopes in the human melanoma antigen gp100 following patient immunization with synthetic peptides. Cancer Res 1996, 56:4749-4757.

59. Salgaller ML, Afshar A, Marincola FM, et al.: Recognition of multiple epitopes of the human melanoma antigen gp100 by peripheral blood lymphocytes stimulated in vitro with synthetic peptides. Cancer Res 1995, 55:4972-4977.

60. Cormier JN, Sal galler ML, PrevetteT, et al.: Enhancement of cellular immunity in melanoma patients immunized with a peptide from MART-1/Melan A. 1997 Cancer J Sci Am 1997, 3:37-44.

61. Parkhurst MR, Salgaller ML, Southwood S, et al.: Improved induction of melanoma reactive CTL with peptides from melanoma antigen gp100 modified at HLA-A0201 binding residues. J Immunology 1996, 157:2536-2548.

62. Wang F, Bade E, Kuniyoshi C, et al.: Phase I trial of a MART-1 peptide vaccine with incomplete Freund's adjuvant for resected high-risk melanoma. Clin Cancer Res 1999, in press.

63. Valmori D, Fonteneau JF, Lizana CM, et al.: Enhanced generation of specific tumor-reactive CTL in vitro by selected Melan-A/MART-1 immunodominant peptide analogues. J Immunol 1998, 160:1750-1758.

64. - Rosenberg SA, YangJC, Schwartzentruber DJ, et al.: Immunologic and therapeutic evaluation of a synthetic peptide vaccine for the treatment of patients with metastatic melanoma. $N$ ature $M$ ed 1998, 4:321-327.

Report of an extensive series of pilot trials in which patients with metastatic melanoma were immunized with different melanoma peptides with an adjuvant and had detectable immune responses especially when a substituted gp100 peptide was used in the vaccine When high-dose interleukin 2 (IL-2) was added to the gp100 melanoma vaccine regimen, significantly increased antitumor responses were observed compared with IL-2 alone

65. Rosenberg SA, Lotze MT, YangJC, et al.: Experience with the use of high-dose interleukin-2 in the treatment of 652 cancer patients. Ann Surg 1990, 210:474-480.

66. Parkinson DR, AbramsJS, Wiernik PH, et al.: Interleukin-2 therapy in patients with metastatic melanoma: a phase II study. J Clin Oncol 1990, 8:1650-1659.

67. Lewis JJ, Janetski S, Wang S, Williams S, et al.: Phase I trial of vaccination with tyrosinase peptide plus Q S-21 in melanoma. Proc ASCO 1998, 17:1650A.

68. Mukherji B, Chakraborty NG, Yamasaki S, et al.: Induction of antigen-specific cytolytic $T$ cells in situ in human melanoma by immunization with synthetic peptide-pulsed autologous antigen presenting cells. Proc N atl Acad Sci USA 1995, 92:8079-8082. 
69. Gajewski TF, Fallarino F, Vogelzang N, et al.: Effective antigenspecific vaccination without dendritic cells (DC): a phase I study of immunization with MAGE-3 or Melan-A peptidepulsed autologous PBMC plus rhulL-12. ProC ASCO 1999, 18:539A.

70. Rosenberg SA, Zhou Y, YangJC, et al.: Immunizing patients with metastatic melanoma using a recombinant adenovirus encoding MART-1 or gp100 melanoma antigens. J N atl Cancer Inst 1998, 90:1894-1900.

71. Steinman RM: The dendritic cell system and its role in immunogenicity. Annu Rev Immunol 1991, 9:271-290.

72. Van Voorhis WC, Hair SL, Steinman RM, et al.: Human dendritic cells:enrichment and characterization from human blood. J Exp M ed 1982, 155:1172-1183.

73. Steinman RM, Witmer MD: Lymphoid dendritic cells are potent stimulators of the primary mixed leukocyte response in mice. Proc $N$ atl Acad Sci U S A 1978, 75:5132-5136.

74. Inaba K, Metlay J.P, Crowley MT, et al.: Dendritic cells pulsed with protein antigens in vitro can prime antigen-specific, MHC-restricted T cells in situ. J Exp M ed 1990, 172:631-640.

75. Inaba K, Young JW, Steinman RM: Direct activation of CD8+ cytolytic T lymphocytes by dendritic cells. J Exp M ed 1987, 166:182-194.

76. Macatonia SE, Taylor PM, Knight SC, et al.: Primary stimulation by dendritic cells induces antiviral proliferative and cytotoxic T responses in vitro. J Exp M ed 1988, 169:1255-1264.

77. Sallustro F, Lanzavecchia A: Efficient presentation of soluble antigen by cultured human dendritic cells is maintained by granulocyte/macrophage colony-stimulating factor plus interleukin 4 and downregulated by tumor necrosis factor. J Exp M ed 1994, 179:1109-1111822.

78. Romani N, Gruner S, Brang D, et al.: Proliferating dendritic cell progenitors in human blood. J Exp M ed 1994, 180:83-93.

79. Van Elsas A, Van der Burg SH, Van Der MinneCE, et al.: Peptidepulsed dendritic cells induce tumoricidal cytolytic $T$ cells from healthy donors against stable HLA-A0201 binding peptides from Melan A/MART-1 self antigen. Eur J Immunol 1996, 26: 1683-1689.

80. - NestleFO, Alijagic S, Gilliet M, et al.: Vaccination of melanoma patients with peptide- or tumor lysate-pulsed dendritic cells. N ature M ed 1998, 4:378-332.

The first report of clinical and immune responses in patients treated with dendritic cells derived from human peripheral blood and grown ex vivo with interleukin-4 and granulocyte-macrophage colonystimulating factor.

81. Chen YT, Stockert E, Jungbluth A, et al.: Serological analysis of Melan-A (MART-1), a melanocyte-specific protein homogeneously expressed in human melanomas. Proc $\mathrm{N}$ atl Acad Sci U S A 1996, 93:5915-5919.

82. Marincola FM, Hijazi YM, Fetsch P, et al.: Analysis of expression of the melanoma-associated antigens MART-1 and gp100 in metastatic melanoma cell lines and in in situ lesions. J Immunother 1996, 19:192-205.
83. Kageshita T, Kawakami Y, Hirai S, Ono T: Differential expression of MART-1 in primary and metastatic melanoma Iesions. J Immunother 1997, 20:460-465.

84. Fetsch PA, Cormier J, Hijazi YM: Immunocytochemical detection of MART-1 in fresh and paraffin-embedded malignant melanomas. J Immunother 1997, 20:60-64.

85. de Vries TJ, Fourkour A, Wobbes T, et al...Heterogeneous expression of immunotherapy candidate proteins gp100, MART-1, and tyrosinase in human melanoma cell lines and in human melanocytic lesions. Cancer Res 1997, 57:3223-3229.

86. Dalerba P, Ricci A, Russo V, et al.: High homogeneity of MAGE, BAGE, GAGE, tyrosinase and Melan-A/MART-1 gene expression in clusters of multiple simultaneous metastases of human melanoma: implications for protocol design of therapeutic antigen-specific vaccination strategies. Int J Cancer 1998, 77:200-204.

87. Cormier JN, Abati A, Fetsch $\mathrm{P}$, et al.: Comparative analysis of the in vivo expression of tyrosinase, MART-1/Melan-A, and gp100 in metastatic melanoma lesions: implications for immunotherapy. J Immunother 1998, 21:27-31.

88. Maeurer MJ, Gollin SM, Martin D, et al.: Tumor escape from immune recognition: lethal recurrent melanoma in a patient associated with downregulation of the peptide transporter protein TAP-1 and loss of expression of the immunodominant MART-1/Melan-A antigen. J Clin Invest 1996, 98:1633-1641.

89. Jaeger E, Ringhoofer M, Karbac J, et al.: Inverse relationship of melanoma differentiation antigen expression in melanoma tissues and CD 8+ cytotoxic $T$ cell responses: evidence for immunoselection of antigen loss variants in vivo. Int J Cancer 1996, 66:470-476.

90. Pittet MJ, Valmori D, Dunbar PR, et al.: High frequencies of naïve Melan A/MART-1-specific CD 8+ T cells in a large proportion of human histocompatibility leukocyte antigen (HLA)-A2 individuals. J Exp M ed 1999, 190:705-715.

91. - Lee PP, Yee C, Savage PA, et al.: Characterization of circulating T cells specific for tumor-associated antigens in melanoma patients. N ature M ed 1999, 5:677-685.

The first report of the use of tetramer technology to detect and anal yze circulating antigen-specific T-cells in melanoma patients in which significant defects in antigen-driven signal transduction were described.

92. Finke JH, Zea AH, Stanley J, et al.: Loss of T-cell receptor chain and p56Ick in T-cells infiltrating human carcinomas. 1993 Cancer Res 1993, 53:5613-5618.

93. Nakagomi H, Petersson M, Magnusson I, et al.: Decreased expression of the signal-transducing chains in tumor infiltrating cells and NK cells of patients with colorectal carcinoma. Cancer Res 1993, 53:5610-5612.

94. Zea $\mathrm{AH}$, Brendan CD, Longo DL, et al.: Alterations in T cell receptor and signal transduction molecules in melanoma patients. Clin Cancer Res 1995, 1:1327-1335. 